\title{
Preoperative Staging of Rectal Tumors: What is the Most Optimal Staging Method?
}

\author{
Regina G.H. Beets-Tan Geerard L. Beets \\ Department of Radiology and Surgery, University Hospital Maastricht, The Netherlands
}

For a better local control rectal cancer patients are nowadays stratified for a more individual treatment according to risks. Besides optimal surgical treatment - by total mesorectal excision (TME) - neoadjuvant radiotherapy with or without chemotherapy has in trials proven its efficacy in reducing the local recurrence rate $[1,2]$. These trials also showed that 3 main risk factors exist for local recurrence of rectal cancer: an involved circumferential resection margin (CRM), involved nodes, and tumors located at the lower rectum. $\mathrm{N}+$ patients as shown by the subgroup analysis of the Dutch TME trial benefited most from preoperative radiotherapy with significant reduction of the local recurrence rate for stage III disease from 21.5 to $11.2 \%$ at 5 years, while at the same time it was shown that a sort-course preoperative radiation scheme is not sufficient for good local control in these high-risk patients [1]. In locally advanced tumors neoadjuvant chemoradiation has shown to effectively downsize and often downstage the tumor leading to new challenging questions regarding whether after tumor shrinkage an extensive resection should still be performed. On the other hand, for patients with stage I disease the TME trial has shown that preoperative radiotherapy had a negligible effect on local recurrence rate (surgery only: $1.7 \%$ vs. radiation plus surgery: $0.4 \%$ ), so preoperative radiotherapy could even be omitted in this group.

Stratification of patients into a tailored treatment is only possible when an accurate selection tool exists that can predict the most relevant risk factors. It is now proven that magnetic resonance imaging (MRI) is the best technique for predicting those patients with an anticipated involved CRM [3,4]. Therefore MRI is being recommended in many European guidelines for rectal cancer treatment as the first-choice staging method. MRI, however, is more expensive, not as widely available as computed tomography (CT) and certainly more dependent on the readers' expertise than the more standardized CT. Dinter et al. [5] have managed to prospectively study side by side staging with MRI, CT, and endorectal ultrasound (EUS). The authors focused on 2 of the remaining risk factors: the $\mathrm{T}$ and $\mathrm{N}$ stage.

Which lessons can we learn from their study? First of all this study shows that each modality, no matter how accurate, has its shortcomings and knowledge of these is of utmost importance for those clinicians involved in rectal cancer treatment. To know the limits of the performance of each modality would lead to an improved staging for each individual patient, allowing the clinician and radiologist to be flexible to choose between the different methods, depending on the clinical question that needs to be solved. In other words, a multimodality staging algorithm is preferred above a single modality algorithm.

The T staging results between the 3 tested imaging methods were comparable, overstaging and understaging errors occurring as much with EUS as with planar imaging. The results again tell us that although EUS is the only method that visualizes each individual layer of the rectal wall and is the best method to distinguish between $\mathrm{T} 1$ and $\mathrm{T} 2$, even in experienced hands it shows the same pitfall of difficult distinction between T2 and borderline desmoplastic T3. Microscopic tumor nests within these desmoplastic reactions remain beyond the detection rate of any anatomical imaging method, no matter how high its resolution. This study also proves that EUS is limited for the advanced tumors in that its field of view (FOV) is too narrow. In the bulky tumors this narrow FOV hampers an overview of the (extra) mesorectal field, leading to lack of visualization of regions at far distance from the probe. Not only does this lead to underestimation of the tumor size and nonvisualization of the circumferential margins but it also leads to understaging of the nodal status, the latter obviously being unacceptable for patients with superficial tumors who are considered for local (transanal) excision.

\begin{tabular}{|c|c|}
\hline KARGER & (c) 2008 S. Karger GmbH, Freiburg \\
\hline $\begin{array}{l}\text { Fax +497614520714 } \\
\text { E-mail Information@Karger.de } \\
\text { www.karger.com }\end{array}$ & $\begin{array}{l}\text { Accessible online at: } \\
\text { www.karger.com/onk }\end{array}$ \\
\hline
\end{tabular}

Regina G.H. Beets-Tan, MD, PhD

Department of Radiology

University Hospital Maastricht

P.O. Box 5800, 6202 AZ Maastricht, The Netherlands

Tel. + 31433876910 , tel + 31433876909

E-mail R.beetstan@radiology.azm.nl 
A word of caution is necessary. Although this study is a unique side-by-side comparison of the 3 most relevant staging methods in rectal cancer, definite conclusions can only be drawn from prospective studies with larger numbers of patients. Furthermore, it has to be mentioned that the MR technique that is used is neither the optimal nor the standard method. The low MR field strength with inherent low spatial and contrast resolution consequently requires the use of 2 coils, both endorectal and phased array external coil, while it is known that at $1.5 \mathrm{~T}$ only an external phased array coil is sufficiently accurate for staging rectal cancer. One should be aware that an endorectal coil technique not only is more invasive and thus less patient friendly, but also difficult to position in patients with high and/or stenosing tumors.

Also the endorectal coil MR technique as well as the hydro CT technique are not preferred as the first choice of techniques in rectal cancer staging. It is known that stretch of the bowel wall by the luminal coil or by intraluminal rectal filling can lead to underestimation of the distance from the tumor to the mesorectal fascia in almost half of the patients especially with low-lying tumors, consequently leading to a significant number of false positive predictions of involved circumferential resection margins and a significant proportion of patients receiving an intensive neoadjuvant treatment while a short course would do. While overtreatment is of less impact than undertreatment this high false positive rate should be avoided, when there is a way to do so. When the use of less optimal protocols would lead to overtreatment in a significant number of patients this would only derive us from the main goal of preoperative stratification by imaging: allowing a tailored treatment for the individual rectal cancer patient that combines the best local control with the lowest treatment morbidity and lowest cost. Therefore knowledge of the optimal protocol for each imaging staging tool is as important as knowledge of the limitations of each treatment modality.

\section{References}

1 Kapiteijn E, Marijnen CA, Nagtegaal ID, et al.: Preoperative radiotherapy combined with total mesorectal excision for resectable rectal cancer. N Engl J Med 2001;345:638-646

2 Sauer R, Becker H, Hohenberger W, et al.: Preoperative versus postoperative chemoradiotherapy for rectal cancer. N Engl J Med 2004;351:1731-1740.

3 MERCURY Study Group: Extramural depth of tumor invasion at thin-section MR in patients with rectal cancer: results of the MERCURY study. Radiology 2007;243:132-139.

4 Beets-Tan RG, Beets GL, Vliegen RF, Kessels AG, Van Boven H, De Bruine A, von Meyenfeldt MF, Baeten CG, van Engelshoven JM: Accuracy of magnetic resonance imaging in prediction of tumour-free resection margin in rectal cancer surgery. Lancet 2001;357:497-504.

5 Dinter DJ, Hofheinz RD, Hartel M, Kähler G, Neff KW, Diehl SJ: Preoperative staging of rectal tumors: comparison of endorectal ultrasound, hydro-CT, and high-resolution endorectal MRI. Onkologie 2008;31: DOI: 10.1159/000121359. 